\title{
The enigmatic ribosomal stalk
}

\section{Quarterly Reviews of Biophysics}

\section{cambridge.org/qrb}

\section{Article}

Cite this article: Liljas A, Sanyal S (2018). The enigmatic ribosomal stalk. Quarterly Reviews of Biophysics 51, e12, 1-10. https://doi.org/ $10.1017 / \mathrm{S} 0033583518000100$

Received: 16 September 2018

Revised: 15 October 2018

\section{Key words:}

Ribosomal stalk; bL12; P1/P2; translational GTPases (trGTPases)

\section{Author for correspondence:}

Anders Liljas, E-mail: anders.liljas@

biochemistry.lu.se
Accepted: 31 October 2018

Anders Liljas ${ }^{1}$ and Suparna Sanyal ${ }^{2}$

${ }^{1}$ Department of Biochemistry and Structural Biology, Center for Chemistry and Chemical Engineering, Lund University, Lund, Sweden and ${ }^{2}$ Department of Cell and Molecular Biology, Biomedical Center, Uppsala University, Uppsala, Sweden

\section{Abstract}

The large ribosomal subunit has a distinct feature, the stalk, extending outside the ribosome. In bacteria it is called the L12 stalk. The base of the stalk is protein uL10 to which two or three dimers of proteins bL12 bind. In archea and eukarya P1 and P2 proteins constitute the stalk. All these extending proteins, that have a high degree of flexibility due to a hinge between their N- and C-terminal parts, are essential for proper functionalization of some of the translation factors. The role of the stalk proteins has remained enigmatic for decades but is gradually approaching an understanding. In this review we summarise the knowhow about the structure and function of the ribosomal stalk till date starting from the early phase of ribosome research.

\section{Introduction}

The ribosome translates a messenger RNA (mRNA) into a chain of amino acid residues to produce proteins. In order to proceed at a speed that is required to sustain life translation factors are needed to catalyze the process. Several of these factors bind in complex with GTP molecules and dissociate after GTP hydrolysis. Non-cleavable GTP analogues inhibit the participation of these factors in protein synthesis. These GTP-depending factors can be called translational GTPases (referred as trGTPases). The induction of GTP hydrolysis has for a long time been an important question.

The two subunits of the ribosome in bacteria are usually composed of two large and one smaller RNA molecule and a large number of ribosomal proteins (See the book by Liljas \& Ehrenberg, 2013). The early electron microscopic studies of the ribosomal large subunit identified a prominent stalk-like extension composed of the protein bL12 (Fig. 1) (Boublik et al., 1976; Lake, 1976; Strycharz et al., 1978). Removing protein bL12 has detrimental effects on the functionality of the trGTPases (Kischa et al., 1971; Hamel et al., 1972; Koteliansky et al., 1978; Pettersson and Kurland, 1980; Mohr et al., 2002; Huang et al., 2010).

The organization of the ribosomal stalk proteins in different kinds of ribosomes and their role in relation to the GTP hydrolysis has remained enigmatic for decades but is gradually approaching an understanding.

\section{The L7/L12 stalk}

\section{The protein bL12}

The protein L7/L12 is an acidic protein that was first characterized in Escherichia coli, where its $\mathrm{N}$-terminus can be acetylated (Terhorst et al., 1972). The acetylated form of L12 is referred to as L7; this is rarely seen in other species. A new naming system has been introduced to accentuate whether ribosomal proteins are universal (and get the prefix $u$ ) or occur only in bacteria (prefix b), archaea (a) or (e) for uniquely eukaryotic proteins (Ban et al., 2014). According to this naming system, L7/L12 is now called bL12. For reviews on bL12 see Liljas, 1982, 1991; Gudkov, 1997; Sanyal and Liljas, 2000; Wahl and Möller, 2002; Gonzalo and Reboud, 2003.

\section{The stoichiometry of bL12}

(c) The Author(s) 2018. This is an Open Access article, distributed under the terms of the Creative Commons Attribution licence (http:// creativecommons.org/licenses/by/4.0/), which permits unrestricted re-use, distribution, and reproduction in any medium, provided the original work is properly cited.

\section{CAMBRIDGE UNIVERSITY PRESS}

The primary analysis of the stoichiometry of ribosomal proteins demonstrated that all proteins are present in one copy per ribosome except bL12, which was found in more than two copies per ribosome (Hardy, 1975; Subramanian, 1975). Later chemical crosslinking and small angle X-ray scattering studies showed that bL12 forms dimers (Österberg et al., 1976). Thus the stoichiometry of the protein on the ribosome should always be in multiples of two. In E. coli there are two bL12 dimers (Österberg et al., 1977), but some species (e.g. Deinococcus radiodurans, Thermus thermophilus) can have three dimers of bL12 (Ilag et al., 2005). The dimers of bL12 bind to the ribosome through protein uL10 (Diaconu et al., 2005). The tight pentameric complex (bL12) 4 .uL10 was initially identified as a unique protein (called L8) not only in E. coli (Kaltschmidt and Wittmann, 1969, 1970; Stöffler, 1974) but also in Bacillus stearothermophilus 


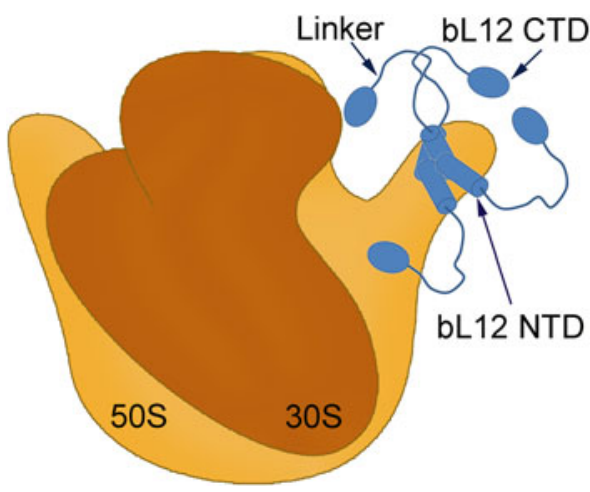

Fig. 1. A symbolic representation of the bacterial ribosome with its small (brown) and large (orange) subunits labelled as the $30 \mathrm{~S}$ and 50S, respectively. Four copies of the protein bL12 are seen on the right-hand side (blue). Each monomer is composed of two domains (bL12 NTD and bL12 CTD) joined by a flexible linker. The bL12 NTD bind to the protein uL10 of the $50 \mathrm{~S}$ subunit while the bL12 CTDs are free to move held only by the linker.

(Cohlberg and Nomura, 1976). It was shown that by mixing bL12 with uL10 in vitro, the 'unique protein', L8, could be formed (Pettersson et al., 1976).

\section{Ribosomal location and basic structure}

Electron microscopy showed that bL12 (formerly L7/L12) forms a stalk-like extension, the so-called L7/L12 stalk on the right-hand side of the large subunit when viewed from the subunit interface (Fig. 1; Boublik et al., 1976; Strycharz et al., 1978). This elongated protein has two structured domains: N-terminal domain (NTD) and C-terminal domain (CTD) interconnected by a highly flexible 'hinge' segment. The NTDs form the tight dimer and can be seen closely associated with the C-terminal long $\alpha 8$ helix of the protein uL10 (Diaconu et al., 2005). Due to the flexible nature of the hinge, the CTDs could be crosslinked to various locations on the ribosome (Traut et al., 1995; Dey et al., 1998). The flexibility explains why the bL12 stalk is poorly, if at all, visible in cryo-EM or crystallographic structures (Agrawal et al., 1999; Ban et al., 2000; Yusupov et al., 2001; Valle et al., 2003; Harms et al., 2008; Gao et al., 2009; Schmeing et al., 2009; Voorhees et al., 2010; Chen et al., 2013; Lin et al., 2015).

\section{The structure of the bL12 dimer}

The bL12 dimer structures have been solved using X-ray crystallography and NMR (Fig. 2) (Wahl et al., 2000; Bocharov et al., 2004; Christodoulou et al., 2004). These structures clearly depict the structural features of different domains of bL12 and the basis of their dimerization. Additionally, they also demonstrate how the flexible hinge leads to different extended and compact conformations of the bL12 dimer.

The NTD of the bL12 monomer is 35 amino acid long in Escherichia coli and composed of two $\alpha$-helices $(\alpha 1$ and $\alpha 2$; Bocharov et al., 1996; 1998). The helices of the NTDs form a hook around its corresponding partner to form the bL12 dimer. In a bL12 dimer, the monomers are arranged in an anti-parallel fashion with respect to each other (Fig. 2; Sanyal and Liljas, 2000; Wahl et al., 2000; Bocharov et al., 2004).

The CTD of bL12 (residue number 52-120 in E. coli) was the first component of the ribosome for which the crystal

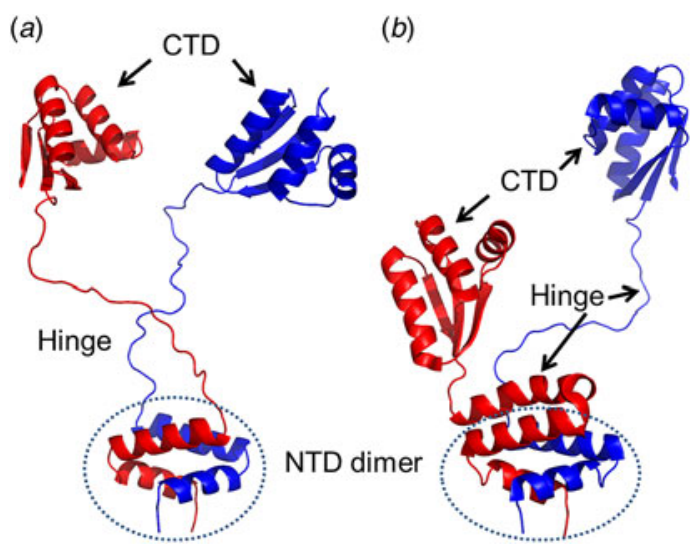

Fig. 2. Ribbon diagrams illustrating different conformations of the bL12 dimer. $a, b-$ The conformations of the bL12 dimer from NMR (Bocharov et al., 2004; PDB ID: 1RQT and $1 \mathrm{RQS}$ ) showing both hinges extended $(a)$ and one in extended and one in a compact state $(b)$. The different domains are marked as CTD (C-terminal domain), NTD dimer (N-terminal domain dimer) and hinge.

structure was determined at atomic resolution (Leijonmarck and Liljas, 1987; Leijonmarck et al., 1980). It has a globular structure with a conserved, positively charged surface, which interacts with the trGTPases (Leijonmarck and Liljas, 1987; Wahl et al., 2000; Bocharov et al., 2004). Two $\alpha$-helices ( $\alpha 4$ and $\alpha 5$ ) of the bL12 CTDs constitute the universal translation factor interaction sites (Savelsbergh et al., 2005; Helgstrand et al., 2007).

NTD and CTD of bL12 are joined by a flexible link or hinge involving residues 36-51 (Bushuev et al., 1989; Bocharov et al., 2004; Mulder et al., 2004). The length and composition of the bL12 hinge, rich in alanyl and glycyl resides, varies considerably between different organisms (Liljas et al., 1986; Bushuev et al., 1989). The hinge is the source of the flexibility of bL12 proteins. From NMR studies it has been suggested that this hinge may be fully extended. Alternatively, it may also transiently form an $\alpha$-helix (Fig. 2a, b) (Bocharov et al., 2004). In the x-ray structure of bL12 from T. maritima (Sanyal and Liljas, 2000; Wahl et al., 2000), where the crystallographic asymmetric unit contains two complete bL12 and two NTDs, both the hinges $(\alpha 3)$ were seen to adopt the compact helical conformations.

NMR spectra of large subunits or whole ribosomes show that bL12 CTD behaves, as if it would be essentially free in solution (Tritton, 1980; Gudkov et al., 1982; Cowgill et al., 1984; Bushuev et al., 1989; Mulder et al., 2004; Bernadó et al., 2010). Furthermore, NMR spectra of bL12 in 50S subunits or 70S ribosomes indicate that only two of the four hinge regions are mobile (Christodoulou et al., 2004; Mulder et al., 2004). It is not known whether the flexible bL12 hinges belong to the same or separate dimers (Sanyal and Liljas, 2000).

\section{The interactions of bL12 with uL10}

The two or three NTD dimers of bL12 (Ilag et al., 2005) all bind to the C-terminal helix $(\alpha 8)$ of protein uL10 (Fig. 3; Diaconu et al., 2005). This helix has a short sequence motif that repeats twice or three times. Each such motif can bind a dimer of bL12. From investigations of the bL12 dimer (Bocharov et al., 1996; Mulder et al., 2004) or the pentameric L8 complex (Bocharov et al., 1998) it is clear that the bL12 CTDs neither interact with each other nor with their NTDs. 


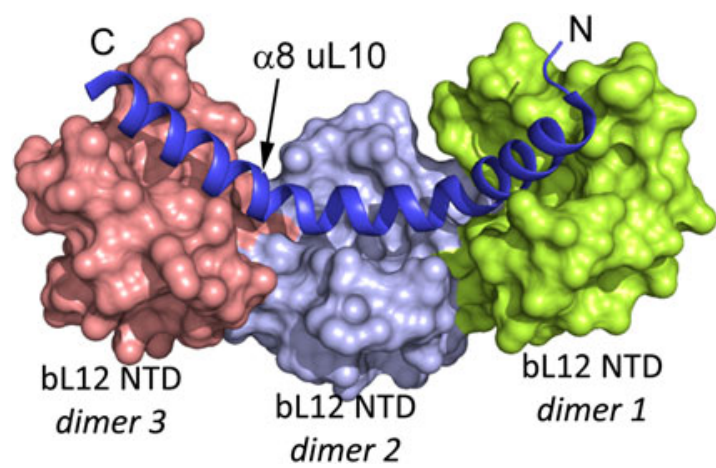

Fig. 3. The structure of three dimers of bL12 NTDs bound to $\alpha 8$ of uL10 (blue) extracted from PDB: 1ZAX.

\section{The 'stalk' on archaeal or eukaryotic ribosomes}

\section{The $P$ proteins}

The proteins corresponding to bacterial bL12 and uL10 in eukaryotes and archaea can be phosphorylated and are therefore called P-proteins (Zinker and Warner, 1976). The protein P0 corresponds to uL10 (Wool et al., 1991). Its N-terminal part is an orthologue of the bacterial form of uL10 (Shimmin et al., 1989). In eukarya there are two related proteins, eP1 and $\mathrm{eP} 2$, which functionally correspond to bL12 in that they bind to the long C-terminal helix of $\mathrm{uL} 10$ as two heterodimers (Tchorzewski et al., 2000a; Maki et al., 2007) to form the bL12 corresponding stalk of the large subunit (Uchiumi et al., 1987; for a review see Liljas, 1991). eP1/eP2 have no sequence similarity with bL12 but share the acidic nature. In archaea, there is only one protein related to eP1 and eP2. It is called aP1. Like in eukaryotes, the archaeal uL10 can bind three dimers of aP1. In addition, the C-terminal region of $\mathrm{P} 0$ in both archaea and eukaryotes contains an extension of about 30 residues identical with the C-terminal residues of P1 and P2 (Santos and Ballesta, 1995).

The eP1 and eP2 have NTDs of about 70 residues followed by a flexible region of about 30 amino acid residues (Wool et al., 1991; Bailey-Serres et al., 1997; Tchorzewski, 2002). This hinge-region is acidic and highly variable in length and amino acid sequence but is rich in alanyl and glycyl residues. The C-termini of all $\mathrm{P}$ proteins has a conserved stretch of 10-13 residues. Several protein kinases can phosphorylate a conserved seryl residue in this region (Ballesta et al., 1999). Like for bL12, the C-terminal regions of the eP1/eP2-proteins interact with trGTPases (Bargis-Surgey et al., 1999). Similar to bL12, the NTDs of aP1 in archaea and $\mathrm{eP} 1$ and $\mathrm{eP} 2$ in eukaryotes are involved in the dimerization (Fig. 4) as well as binding to uL10 (Ballesta and Remacha, 1996). The mode of dimerization involving helical NTDs is more complex in archaea and eukaryotes than in bacteria and involves four $\alpha$-helices from each molecule than two $\alpha$-helices in bacteria (Fig. 4).

In yeast, there are four variants of the $\mathrm{P}$ proteins: $\mathrm{P} 1 \mathrm{~A}, \mathrm{P} 1 \mathrm{~B}$, $\mathrm{P} 2 \mathrm{~A}$ and $\mathrm{P} 2 \mathrm{~B}$. P1A forms a heterodimer with $\mathrm{P} 2 \mathrm{~B}$ and $\mathrm{P} 2 \mathrm{~A}$ forms a heterodimer with P1B (Tchorzewski et al., 2000b; Guarinos et al., 2001). In plants, there is one additional P protein, P3 (Bailey-Serres et al., 1997). If all P-proteins are deleted, the ribosomes do not function. If only the $\mathrm{P} 1$ and $\mathrm{P} 2$ proteins are missing, the ribosome is able to support protein synthesis with the C-terminally extended uL10 alone (Santos \& Ballesta, 1994; Remacha et al., 1995), but a different subset of mRNAs is translated. However, if the $\sim 30$ amino acid long C-terminal residues of $\mathrm{uL} 10$ corresponding to the C-terminus of $\mathrm{P} 1 / \mathrm{P} 2$ are also removed from $\mathrm{uL} 10$, the ribosome is no more functional.

\section{The structure of the P-protein stalk}

The structures of $\mathrm{uL10}$ and the stalk complex from the archaeal species have been determined at high resolution (Kravchenko et al., 2010; Naganuma et al., 2010). The latter structure contains $\mathrm{uL} 10$ with three dimers of the N-termini of aP1. The structure of archaeal uL10 is closely similar to bacterial uL10, whereas the NTDs of aP1 are each composed of four $\alpha$-helices in two layers. The dimer interface is formed by the two N-terminal helices (Fig. 4).

In all species, this stalk of the ribosome is long and flexible. The archaeal uL10 C-terminal helix binds over the 2-fold symmetry axis of the dimer of aP1, like in the uL10-bL12 interaction. The first and the fourth helix of aP1 interact almost orthogonally with the long helix of uL10 (Fig. 5). The possible arrangement of the archaeal $\mathrm{P}$ proteins in the ribosome will not be much different from the situation in the bacterial ribosome. One difference is that the $\mathrm{C}$-terminal domain of the $\mathrm{P}$ proteins is smaller and that there is a C-terminal extension of uL10 of the same nature as the C-terminal domain of the P1 and P2 proteins.

\section{The trGTPases and their interactions with bL12 on the ribosome}

\section{The trGTPases}

There are a number of trGTPases involved in protein synthesis (Table 1).

The trGTPases catalyze different steps of translation. These proteins, in their GTP-bound states catalyze different steps of translation on the ribosome. Further, they hydrolyze the bound GTP molecule and dissociate from the ribosome. The different roles of the trGTPases necessitate their different structures (Fig. 6), but they all have two domains in common, the GTP binding domain ( $\mathrm{G}$ domain), normally at the $\mathrm{N}$-terminus and the
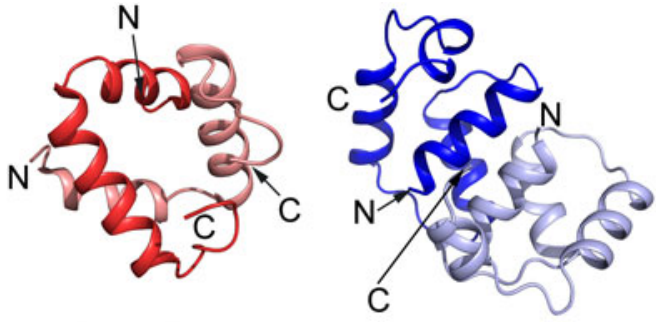

Archaea

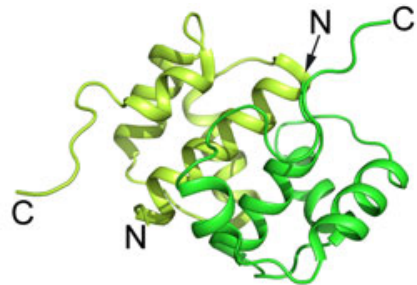

Eukarya
Fig. 4. Comparison of the structure of the NTD dimer of bacterial bL12 with archaeal protein aP1 and eukaryotic eP1/P2 proteins adopted from the PDBs $1 R Q T, 3 A 1 Y$, and $2 \mathrm{LBF}$, respectively. While the archaeal and eukaryotic domains are similar, in comparison with the bacterial domain it is difficult to identify a structural similarity apart from the helical structure. 
Fig. 5. Structure of the archaeal stalk complex (PDB: 3A1Y). uL10 is colored blue, while three aP1 dimers bound to the spine helices are colored in salmon, light blue and lemon. The aP1 dimers bind to the long C-terminal helix of uL10.

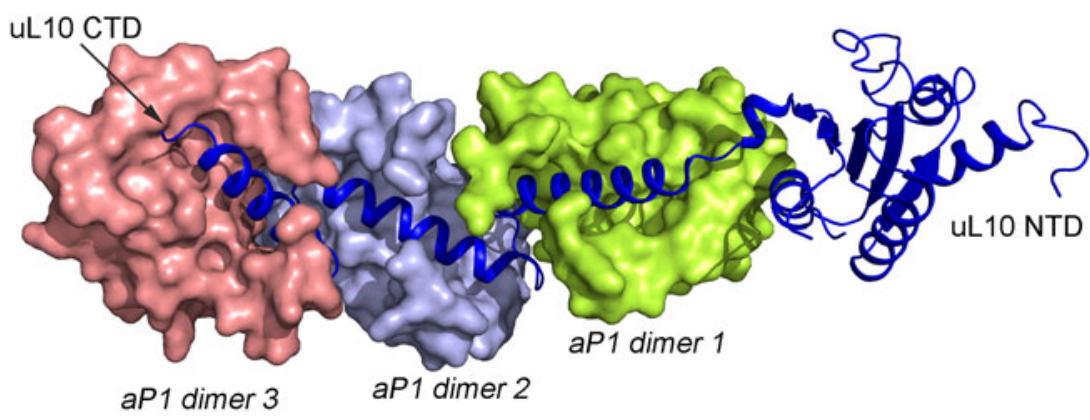

Table 1. Some trGTPases involved in protein synthesis on the ribosome

\begin{tabular}{|c|c|c|}
\hline Bacteria & $\begin{array}{l}\text { Archaea and } \\
\text { Eukarya }\end{array}$ & Functional role \\
\hline bIF2 & elF5B & Association of large and small subunits during initiation \\
\hline bEF-Tu & eEF-1A & Carrier of aminoacyl-tRNA to the decoding site \\
\hline bSelB & eEF-Sec & Carrier of Se-Met-tRNA to the decoding site \\
\hline bEF-G & eEF2 & $\begin{array}{l}\text { Translocation of peptidyl-tRNA from A-site to P-site and recycling of ribosomal subunits together with the ribosome } \\
\text { recycling factor, RRF }\end{array}$ \\
\hline bRF3 & eRF3 & $\begin{array}{l}\text { Removal of release factors } 1 \text { and } 2 \text { from the ribosome. The bacterial protein is completely different from the archaeal } \\
\text { and the eukaryotic proteins. }\end{array}$ \\
\hline $\begin{array}{l}\text { bLepA, } \\
\text { EF4 }\end{array}$ & mtLepA, cpLepA & Back translocation? Ribosome biogenesis? \\
\hline BipA & TypA & Ribosome biogenesis? \\
\hline Tet-O/M & & Removal of tetracycline causing resistance \\
\hline
\end{tabular}

subsequent domain (domain II). Two of the trGTPases, EF-G and RF3, have an insert in the G-domain called $G^{\prime}$ domain (Fig. 6). EF4 and BipA are paralogs of EF-G, but among other differences, they lack the $G^{\prime}$ domain. For further structural and functional comparisons see Liljas and Ehrenberg (2013); Heller et al. (2017); Gibbs and Fredrick (2018).

\section{The binding of trGTPases to the ribosome}

The trGTPases in complex with GTP bind to overlapping sites on the ribosome (Fig. 7) (Heimark et al., 1976). The two common domains of the factors, G and II, interact with the ribosome in similar ways. These interactions, identified by cryo-EM and crystallography, show that the G-domains interact primarily with the sarcin-ricin loop (SRL) and the GTPase activating region (GAR) of the large subunit at the base of the bL12 stalk. The binding sites include RNA-helices H43 and H44 as well as proteins uL11 and uL6 (Gao et al., 2009; Schmeing et al., 2009). The anticodon-stem-loop of the tRNA in the ternary complex, EF-Tu-GTP-aa-tRNA interacts with the decoding region of the small subunit and so does the domain IV of EF-G. IF2, on the contrary, interacts initially only with the $30 \mathrm{~S}$ subunit. When the subunits associate IF2 makes substantial contacts with the GAR of the 50S subunit. RF3 interacts mostly with the 50S subunit in a similar fashion as EF-Tu (Gao et al., 2007; Pallesen et al., 2013).
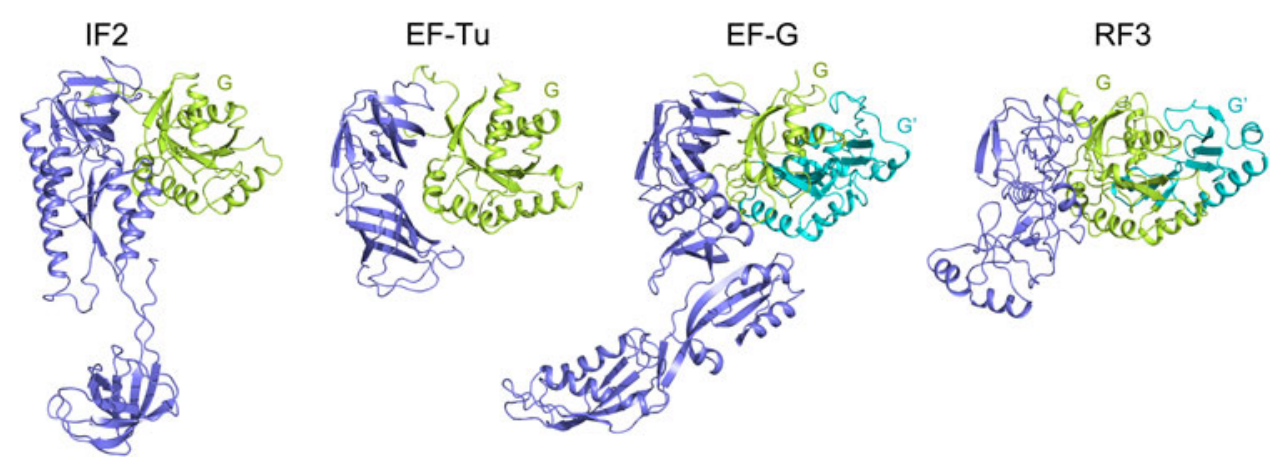

Fig. 6. The structure of the major trGTPases showing $G$ and $G^{\prime}$ domains in green and cyan. The structures are adopted from the following PDBs: IF2 (PDB: $3 J C N$ ), EF-Tu (PDB: 4PC7), EF-G (PDB: 4V9O) and RF3 (PDB: 4V89). 

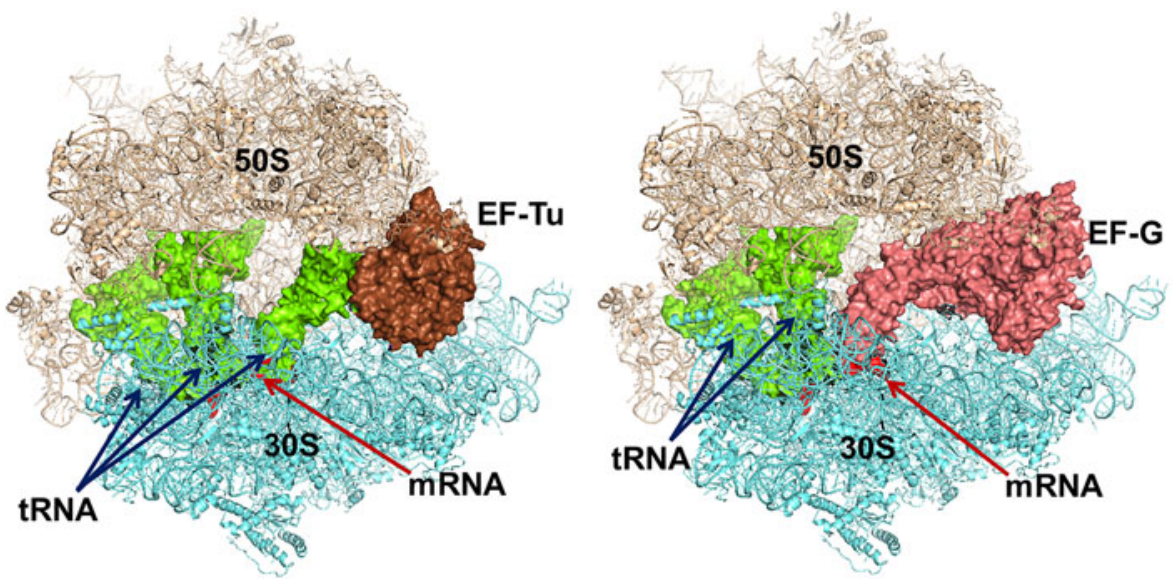

Fig. 7. Left - Elongation factor Tu (EF-Tu) in complex with an aminoacyl tRNA and a GTP analogue bound to the ribosome. Right - Elongation factor $G$ with a GTP analogue bound to the ribosome. The similarity in binding is striking. The structures are adopted from the following PDBs: EF-Tu (PDB: 4V5R), EF-G (PDB: 4V5F).

\section{What induces GTPase activity in the trGTPases?}

The induction of GTPase activity in the trGTPases by the ribosome has been a challenging question. The trGTPases are part of a large family of GTPases where generally a GTPase activating protein (GAP) participates at the right stage and induces GTP hydrolysis. Furthermore, a residue in a loop of the GTPase, the so-called Switch II, has been thoroughly discussed. In most GTPases, this residue is a glutamine. Its ability to function as a general base is severely limited (Langen et al., 1992). The corresponding residue in the trGTPases is a histidine, which would normally be able to function as a general base. However, since the GTPases have a common evolution it is most likely that the mechanism for GTP hydrolysis is the same, and that the histidine has a different role.

Several investigations have focused on the question of whether bL12 could be the ribosomal GAP and whether the histidine in Switch II of the trGTPases could function as a general base. Previously, it was proposed that isolated bL12 could independently stimulate GTP hydrolysis of EF-G on the ribosome unlike EF-Tu (Savelsbergh et al., 2000). Subsequent kinetic studies revealed that depletion of bL12 from the $70 \mathrm{~S}$ ribosome selectively affects stimulation of GTP hydrolysis in both EF-Tu and EF-G under multiple turnovers (Mohr et al., 2002; Savelsbergh et al., 2005) or single turnover condition (Diaconu et al., 2005). However, it was demonstrated that for GTPase stimulation on IF2, bL12 does not play any role (Huang et al., 2010). Also, highresolution structures of EF-Tu and EF-G on the ribosome demonstrated that bL12 is not in contact with the G-domains of the trGTPases (Voorhees et al., 2010; Carlson et al., 2017). Therefore, the precise role of bL12 in the context of GTP hydrolysis by trGTPases remains elusive and awaits further investigation.

For a considerable time, the SRL of the large subunit has also been considered to be an important region of the ribosome for factor interactions. Chemical footprinting showed that both EF-Tu and EF-G protect this loop (Moazed et al., 1988). Crystallographic experiments on EF-Tu bound to the ribosome have made it clear that the phosphate of adenine 2662 in the SRL is critically involved in GTP hydrolysis (Voorhees et al., 2010). It was shown that the phosphate of A2662 hydrogen bonds to His84 in EF-Tu and brings it up against the nucleophilic water molecule that is moved into close proximity of the $\gamma$-phosphate of the GTP molecule. His84 was suggested to act like a general base (Berchtold et al., 1993; Voorhees et al., 2010). However, insightful mechanistic analysis supported by in

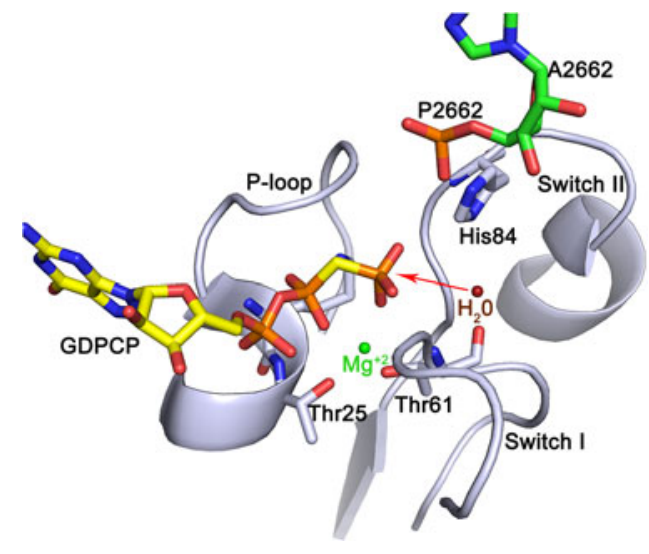

Fig. 8. A possible mechanism for GTP hydrolysis by the trGTPases. The phosphate of A2662 of SRL accepts a hydrogen bond from His 84 of the switch II of EF-Tu and positions it next to the water molecule close to the $\gamma$-phosphate. Both side chain nitrogens of the histidine are protonated making the histidine positively charged. The water molecule (red) donates a proton to the $\gamma$-phosphate leading to an in-line attack by the hydroxyl ion on the $\gamma$-phosphate (Liljas et al., 2011, Åqvist \& Kamerlin, 2015). The structure was adopted from PDB: 4V5L (Voorhees et al., 2010).

silico modeling and molecular dynamics (MD) simulations suggest that it is more likely that the water molecule, possibly through a second water molecule, donates one proton to the $\gamma$-phosphate and attacks the phosphate by an in-line mode, which leads to the hydrolysis of the $\gamma$-phosphate (Fig. 8; Liljas et al., 2011; Åqvist and Kamerlin, 2015; Mondal and Warshel, 2018). This mechanism is probably universal for the trGTPases. Thus, the phosphate of an adenine in the SRL replaces the GTPase activating protein (GAP) for trGTPases in general.

\section{The role of bL12 in the functions of the trGTPases}

The removal of the CTD of bL12 has the same effect as removing the whole protein on the activity of trGTPases (van Agthoven et al., 1975; Koteliansky et al., 1978). Thus, it was concluded that bL12 interacts with the trGTPases through its CTD. Ribosomes with only one bL12 dimer are partially active (Griaznova and Traut, 2000; Mandava et al., 2012). Ribosomes where one CTD of each bL12 dimer was removed also retain activity (Oleinikov et al., 1998). The removal of all bL12 delays the release of inorganic phosphate from EF-G after GTP hydrolysis (Mohr et al., 2002). If the hinge of bL12 is significantly 
Fig. 9. Illustration of the interaction sites of the four major trGTPases IF2 (a), EF-Tu (b), EF-G (c) and RF3 $(d)$ on the bL12 CTD as identified by NMR mapping (Helgstrand et al., 2007). The top panel represents the ribbon diagram and the bottom panel represents the surface illustration of bL12-CTD. The interaction sites are highlighted in red. (a)
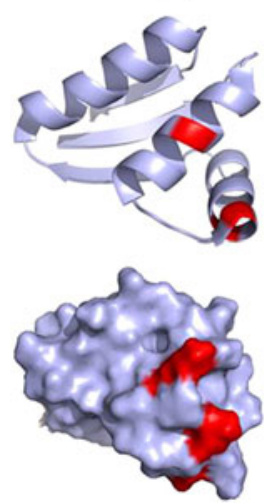

(b)

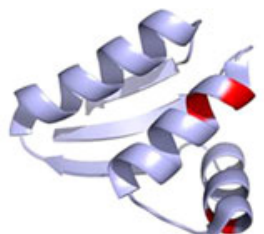

(c)
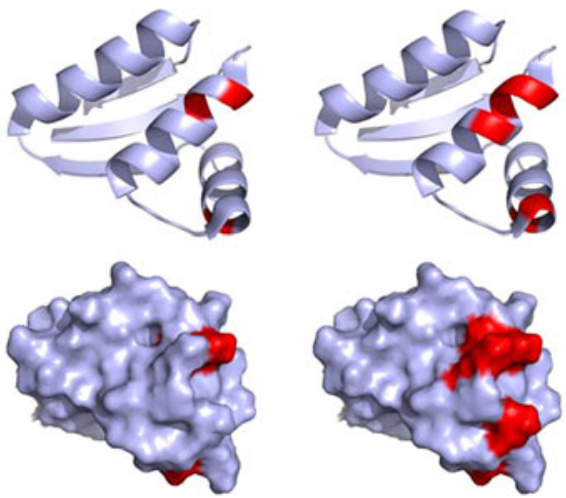

(d)
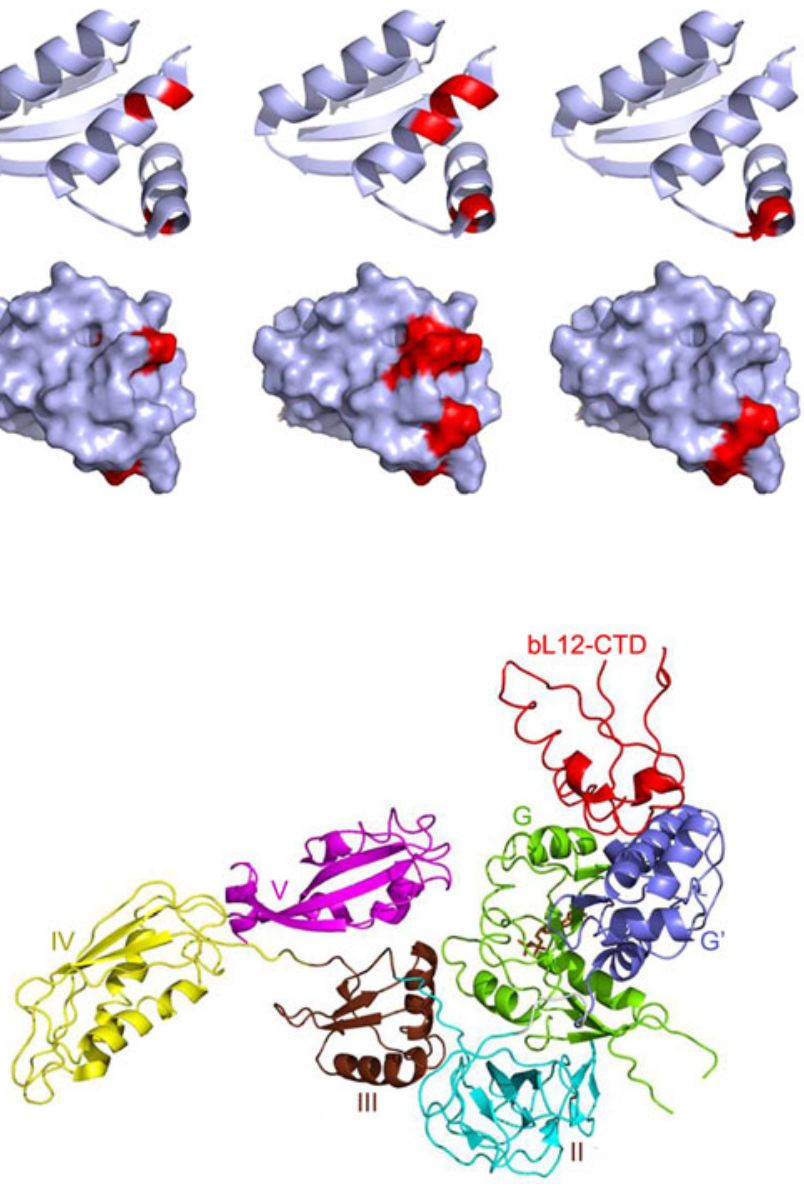

Fig. 10. The interaction of the G' and G domains of EF-G with bL12CTD (red) on T. thermophilus 70S ribosomes (PDB: 4V5F) (Gao et al., 2009).

The main role for the bacterial trGTPase IF2 is to promote efficient ribosomal subunit association. bIF2 forms a preinitiation complex with $30 \mathrm{~S}$ subunit programmed with mRNA and fMet-tRNA $^{\mathrm{fMet}}$. bL12 is needed for rapid factor-dependent subunit association with a $70 \mathrm{~S}$ initiation complex (Huang et al., 2010; Mandava et al., 2012). Cryo-EM and crosslinking have shown that bL12 interacts with the G domain of IF2 (Heimark et al., 1976; Allen et al., 2005). Recently, Ge et al. (2018) analyzed a range of mutants in bL12 and IF2 to identify the sites involved in subunit association (Fig. 11). Mutations of the conserved K65, K70, R73, and K81 on the $\alpha 4-\alpha 5$ helices of bL12 caused a significant defect in subunit association. Similarly, mutations in D506, D508, E522, and E523 of G4-G5 helix of IF2 were found to be deleterious for fast subunit association. It can be easily noticed that the identified residues of bL12 and IF2 are basic and acidic in nature, respectively. Moreover, mutations to oppositely charged amino acids on both bL12 and IF2 caused largest defects, which could be rescued by using charge-swapped mutants on both the interacting partners. This mutational analysis pinpointed the amino acids on bL12 and IF2, which are the molecular determinants for their interaction. In addition, MD simulations identified the details of the interactions of these residues at the molecular level. It suggested several salt-bridge interactions between the bL12 CTD and the G domain of IF2 (Fig. 11). Based on the analysis, one can conclude that charge complementarity is the basis of 


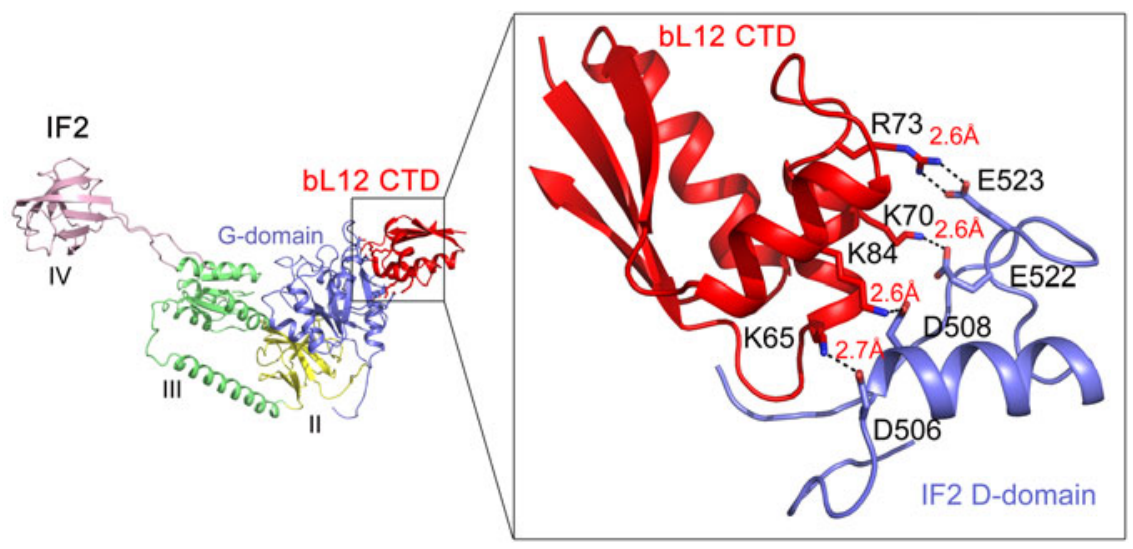

Fig. 11. The interaction of bL12 CTD (red) with IF2 on the $70 \mathrm{~S}$ ribosome as suggested from the mutational studies combined with MD simulations (Ge et al., 2018).

bL12 and IF2 interaction (Ge et al., 2018). Moreover, it can be inferred that bL12 (CTD) on the 50S and IF2 on 30S-preinitiation complex act like recognition partners; their initial interaction leads to the rapid association of the ribosomal subunits. Based on the similarity of the G-domains of the trGTPases it can be speculated that the other trGTPases too interact with bL12 CTD through a similar mechanism based on electrostatic interaction. The available cryo-EM based pictures of bL12 interaction with RF3 (Pallesen et al., 2013) and EF-G (Gao et al., 2009) support that model.

Carlson et al., (2017) have revisited some of the old experiments after significantly improved purification of the experimental components. First of all they used E. coli JE28 ribosomes in which all bL12 proteins carry chromosomally fused C-terminal His-tag (Ederth et al., 2009). With the aid of the His-tag, they ensured that bL12 was completely removed from the ribosomes. They also used an extensive denaturation and renaturation based method for purification of bL12 more extensively. In their study, bL12 did not induce GTPase activity in the isolated EF-G in contrast to what was reported earlier by Savelsbergh et al., 2000. However, the GTPase activity by EF-G on the ribosome requires the presence of bL12. The binding of EF-G in complex with the non-hydrolysable GTP analogue GDPNP to the ribosome was dramatically reduced if bL12 was absent. If the $G^{\prime}$ domain was removed from EF-G the GTPase activity was much reduced and the presence or absence of bL12 in the ribosome did not make any difference. Likewise, the binding of RF3 and IF2 to ribosomes also required the presence of bL12 (Carlson et al., 2017).

\section{The interaction of P-proteins with trGTPases}

In archaeal or eukaryotic translation the P-proteins are essential for the function of the trGTPases. However, all P1/P2 dimers can be removed despite maintaining the GTPase activity. This activity can be maintained by the C-terminus of $\mathrm{uL} 10$ alone. However, the lack of some or all of the P1/P2 dimers leads to losses in fidelity and a different subset of mRNAs is translated (Santos \& Ballesta, 1994; Remacha et al., 1995, Wawiórka et al., 2017). This may suggest a complex role of these proteins but primarily relates to the decoding process where different complexes of EF1A with GTP and aminoacylated tRNAs are tested against the current codon in the A-site. The rate needed for efficient discrimination of non-cognate and near-cognate tRNAs cannot be met by a single C-terminus.

With regard to the CTD of the P-proteins, there has been a lack of information about its structure and interaction with the

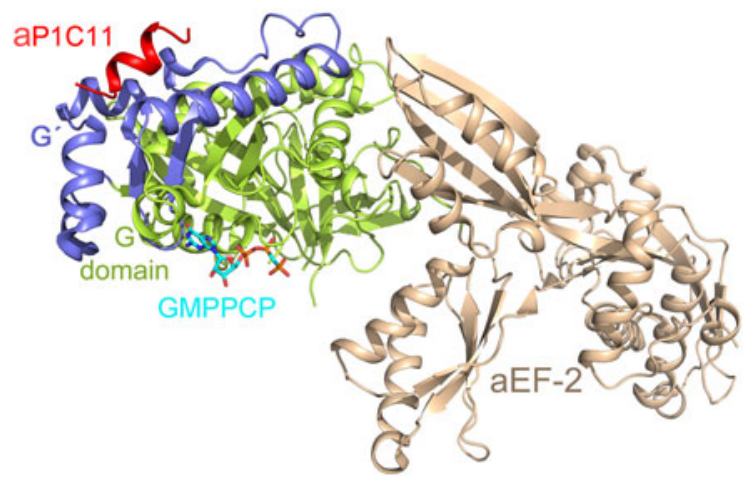

Fig. 12. The C-terminal (C11) peptide of aP1 (red) binds to the archaeal EF2 in a hydrophobic groove between the G (green) and G' (blue) domains (PDB ID: 5H7L) (Tanzawa et al., 2018)

trGTPases. Recently Tanzawa et al. (2018) studied the binding of a C-terminal peptide of aP1 to aEF2. The peptide takes the conformation of a helix, which binds to the G4 and G5 elements of the factor similar to the observations in case of bacterial IF2 (Fig. 12) (Ge et al., 2018). However, in contrast to the charge complementarity-based interaction of bL12-CTD and IF2, the 11 amino acid long C-terminal helix of aP1 (aP1C11) binds to aEF2a in a hydrophobic groove.

\section{Ribosome inhibitory proteins high-jack the ribosomal stalk for binding}

There is a range of ribosome inhibitory proteins (RIP) that generally modify the part of SRL, which is important for the GTPase activity. In some cases, like ricin, a central nucleotide is depurinated and in other cases, like alpha-sarcin, the large ribosomal RNA is cut. The RIPs are evolutionary related, but the sequence conservation is quite low. Interestingly, without any sequence or structural relationship to the trGTPases, the RIPs like the trGTPases benefit in binding to the ribosome from the presence of the P-proteins. The binding is through hydrophobic interactions of the very C-terminal 6-9 residues of the P-proteins (Shi et al., 2016).

\section{Conclusions}

The stalk proteins have very distinctive roles primarily in relation to the trGTPases. However, it remains enigmatic what their actual 
role is in relation to the activation of GTP hydrolysis in trGTPases. There are at least two options generally discussed:

(A) The ribosomal stalk, with multiple arms bearing the C-terminal structures of bL12 or the P-proteins, recruits trGTPases to their binding area on the ribosome by binding and thereby increasing the local concentration of trGTPases. This would, in turn, increase the rate of GTP hydrolysis.

(B) The ribosomal stalk binds the trGTPases on the ribosome and stabilizes their binding to the factor-binding site at the SRL loop on the ribosome thereby increasing the rate of GTP hydrolysis.

The first mechanism (A) is most widely accepted by the ribosome field at present. However, relevant experimental proof supporting this mechanism is still lacking. If the native-like high rate of GTP hydrolysis could be achieved by adding an increasing concentration of the trGTPases with a bL12-depleted ribosome, then this mechanism would be proven true. That would then justify the need for multiple bL12-CTDs for increasing the local concentration of the trGTPases for high GTPase rates. However, the recent study with super-resolution microscopy infers that the four bL12 proteins are essentially saturated with EF-Tu ternary complexes in vivo (Mustafi and Weisshaar, 2018). This observation again speaks in favor of the factor recruitment model.

Experimental evidence for the second mechanism (B) is also scarce. If a high GTPase rate can be achieved by titrating L12CTDs with a bL12-depleted ribosome, it might support model (B). It would mean that the bL12-CTD interaction is important for stable binding of the trGTPases in the vicinity of the SRL and for attaining the correct conformation for GTPase activation. Carlson et al. (2017) found reduced occupancy of EF-G-GDPNP on the ribosome when bL12 is deleted. This result clearly indicates the need of bL12, presumably the CTD, for stable binding of the trGTPases on the ribosome. The case of the initiation factors, bacterial IF2 or archaeal or eukaryotic eIF5B illustrates another aspect of the bL12 CTD (Ge et al., 2018). These factors initially bind to the small subunit and need the stalk proteins for association with the large subunit. These results also clearly support the B alternative. It is also possible that the ribosomal stalk proteins use different or specialized modes of action with respect to different translational GTPases. Its role in the function of other translation factors also remains elusive.

From our discussions of the available literature regarding bL12 it becomes obvious that despite about 50 years of research, the role of the ribosomal stalk remains somewhat enigmatic. On the contrary, the multifaceted research on bL12 and the P proteins has brought us much further into this intriguing story.

Acknowledgements. We are thankful to Dr. Chandra Sekhar Mandava and Dr. Xueliang Ge for helping with the artwork used in this article. SS acknowledges research funding support from Swedish Research Council (2014-4423 and Research Environment Grant 2016-06264), and Knut and Alice Wallenberg Foundation (KAW 2017.0055).

\section{References}

Agrawal RK, Heagle AB, Penczek P, Grassucci RA and Frank J (1999) EF-G-dependent GTP hydrolysis induces translocation accompanied by large conformational changes in the $70 \mathrm{~S}$ ribosome. Nature Structural Biology 6, 643-647.

Allen GS, Zavialov A, Gursky R, Ehrenberg M and Frank J (2005) The cryo-EM structure of a translation initiation complex from Escherichia coli. Cell 121, 703-712.
Åqvist J and Kamerlin S (2015) The conformation of a catalytic loop is central to GTPase activity on the ribosome. Biochemistry 54, 546-556.

Bailey-Serres J, Vangala S, Szick K and Lee CH (1997) Acidic phosphoprotein complex of the $60 \mathrm{~S}$ ribosomal subunit of maize seedling roots. Components and changes in response to flooding. Plant Physiology 114, 1293-1305.

Ballesta JP and Remacha M (1996) The large ribosomal subunit stalk as a regulatory element of the eukaryotic translational machinery. Progress in Nucleic Acid Research and Molecular Biology 55, 157-193.

Ballesta JP, Rodriguez-Gabriel MA, Bou G, Briones E, Zambrano R and Remacha M (1999) Phosphorylation of the yeast ribosomal stalk. Functional effects and enzymes involved in the process. FEMS Microbiological Reviews 23, 537-550.

Ban N, Nissen P, Hansen J, Moore PB and Steitz TA (2000) The complete atomic structure of the large ribosomal subunit at $2 \cdot 4 \AA$ resolution. Science 289, 905-920.

Ban N, Beckmann R, Cate JHD, Dinman JD, Dragon F, Ellis SR, Lafontaine DLJ, Lindahl L, Liljas A, Lipton JM, McAlear MA, Moore PB, Noller HF, Ortega J, Panse VG, Ramakrishnan V, Spahn CMT, Steitz TA, Tchorzewski M, Tollervey MD, Warren AJ, Williamson JR, Wilson D, Yonath A and Yusupov M (2014) A new system for naming ribosomal proteins. Current Opinion in Structural Biology 24, 1-5.

Bargis-Surgey P, Lavergne JP, Gonzalo P, Vard C, Filhol-Cochet $O$ and Reboud JP (1999) Interaction of elongation factor eEF-2 with ribosomal P proteins. European Journal of Biochemistry 262, 606-611.

Berchtold H, Reshetnikova L, Reiser CO, Schirmer NK, Sprinzl M and Hilgenfeldt R (1993) Crystal structure of active elongation factor Tu reveals major domain rearrangements. Nature 365, 126-132.

Bernadó P, Modig K, Grela P, Svergun D, Tchorzewski M, Pons M and Akke M (2010) Structure and dynamics of ribosomal protein L12: an ensemble model based on SAXS and NMR relaxation. Biophysical Journal 98, 2374-2382.

Bocharov EV, Gudkov AT and Arseniev AS (1996) Topology of the secondary structure elements of ribosomal protein L7/L12 from E. coli in solution. FEBS Letters 379, 291-294.

Bocharov EV, Gudkov AT, Budovskaya EV and Arseniev AS (1998) Conformational independence of $\mathrm{N}$ - and $\mathrm{C}$-domains in ribosomal protein L7/L12 and in the complex with protein L10. FEBS Letters 423, 347-350.

Bocharov EV, Sobol AG, Pavlov KV, Korzhnev DM, Jaravine VA, Gudkov AT and Arseniev AS (2004) From structure and dynamics of protein L7/L12 to molecular switching in ribosome. Journal of Biological Chemistry 279, 17697-17706.

Boublik M, Hellmann W and Roth HE (1976) Localization of ribosomal proteins L7/L12 in the 50S subunit of Escherichia coli ribosomes by electron microscopy. Journal of Molecular Biology 107, 479-490.

Brilot AF, Korostelev AA, Ermolenko DN and Grigorieff N (2013) Structure of the ribosome with elongation factor $\mathrm{G}$ trapped in the pretranslocation state. Proceedings of the National Academy of Sciences USA 110, 20994-20999.

Bubunenko MG, Chuikov SV and Gudkov AT (1992) The length of the interdomain region of the L7/L12 protein is important for its function. FEBS Letters 313, 232-234.

Bushuev VN, Gudkov AT, Liljas A and Sepetov NF (1989) The flexible region of protein $\mathrm{L} 12$ from bacterial ribosomes studied by proton nuclear magnetic resonance. Journal of Biological Chemistry 264, 4498-4505.

Carlson MA, Bassam GH, Weis AJ, Colby SB, Shelton CD, Wuerth ME, Walter JD and Spiegel Jr PC (2017) Ribosomal protein L7/L12 is required for GTPase translation factors EF-G, RF3, and IF2 to bind in the GTP state to 70 S ribosomes. FEBS Journal, 284, 1631-1643.

Chen Y, Feng S, Kumar V, Ero R and Gao Y-G (2013) Structure of EF-Gribosome complex in a pretranslocation state. Nature Structural and Molecular Biology 20, 1077-1084.

Christodoulou J, Larsson G, Fucini P, Connell SR, Pertinhez TA, Hanson CL, Redfield C, Nierhaus KH, Robinson CV, Schleucher J and Dobson CM (2004) Heteronuclear NMR investigations of dynamic regions of intact Escherichia coli ribosomes. Proceedings of the National Academy of Sciences USA 101, 10949-10954.

Cohlberg JA and Nomura M (1976) Reconstitution of Bacillus stearothermophilus 50S ribosomal subunits from purified molecular components. Journal of Biological Chemistry 251, 209-221. 
Cowgill CA, Nichols BG, Kenny JW, Butler P, Bradbury EM and Traut RR (1984) Mobile domains in ribosomes revealed by proton nuclear magnetic resonance. Journal of Biological Chemistry 259, 15257-15263.

Datta PP, Sharma MR, Qi L, Frank J and Agrawal RK (2005) Interaction of the $\mathrm{G}$ ' domain of elongation factor $\mathrm{G}$ and the $\mathrm{C}$-terminal domain of ribosomal protein L7/L12 during translocation as revealed by cryo-EM. Molecular Cell 20, 723-731.

Dey D, Bochkariov DE, Jokhadze GG and Traut RR (1998) Cross-linking of selected residues in the $\mathrm{N}$ - and C-terminal domains of Escherichia coli protein L7/L12 to other ribosomal proteins and the effect of elongation factor Tu. Journal of Biological Chemistry 273, 1670-1676.

Diaconu M, Kothe U, Schlunzen F, Fischer N, Harms JM, Tonevitsky AG, Stark H, Rodnina MV and Wahl MC (2005) Structural basis for the function of the ribosomal L7/12 stalk in factor binding and GTPase activation. Cell 121, 991-1004.

Ederth J, Mandava CS, Dasgupta S and Sanyal S (2009) A single-step method for purification of active His-tagged ribosomes from a genetically engineered Escherichia coli. Nucleic Acids Research 37, e15.

Gao H, Zhou Z, Rawat U, Huang C, Bouakaz L, Wang C, Cheng Z, Liu Y, Zavialov A, Gursky R, Sanyal S, Ehrenberg M, Frank J and Song H (2007) RF3 induces ribosomal conformational changes responsible for dissociation of class I release factors. Cell 129, 929-941.

Gao YG, Selmer M, Dunham CM, Weixlbaumer A, Kelley AC and Ramakrishnan V (2009) The structure of the ribosome with elongation factor $\mathrm{G}$ trapped in the posttranslocational state. Science 326, 694-699.

Ge X, Mandava CS, Lind C, Åqvist J and Sanyal S (2018) Complementary charge-based interaction between the ribosomal-stalk protein L7/12 and IF2 is the key to rapid subunit association. Proceedings of the National Academy of Sciences USA 115, 4649-4654.

Gibbs MR and Fredrick K (2018) Roles of elusive translational GTPases come to light and inform on the process of ribosome biogenesis in bacteria. Molecular Microbiology 107, 445-454.

Gonzalo P and Reboud J-P (2003) The puzzling lateral flexible stalk of the ribosome. Biology of the Cell 95, 179-193.

Griaznova O and Traut RR (2000) Deletion of C-terminal residues of Escherichia coli ribosomal protein L10 causes the loss of binding of one L7/L12 dimer: ribosomes with one L7/L12 dimer are active. Biochemistry 39, 4075-4081.

Guarinos E, Remacha M and Ballesta JP (2001) Asymmetric interactions between the acidic $\mathrm{P} 1$ and $\mathrm{P} 2$ proteins in the Saccharomyces cerevisiae ribosomal stalk. Journal of Biological Chemistry 276, 32474-32479.

Gudkov AT (1997) The L7/L12 domain of the ribosome: structural and functional studies. FEBS Letters 407, 253-256.

Gudkov AT, Gongadze GM, Bushuev VN and Okon MS (1982) Proton nuclear magnetic resonance study of the ribosomal protein L7/L12 in situ. FEBS Letters 138, 229-232.

Gudkov AT, Bubunenko M and Gryaznova O (1991) Overexpression of L7/L12 protein with mutations in its flexible region. Biochimie 73, 13871389.

Hamel E, Koka M and Nakamoto T (1972) Requirement of an E. coli 50S ribosomal protein component for effective interaction of the ribosome with $\mathrm{T}$ and $\mathrm{G}$ factors with guanosine triphosphate. Journal of Biological Chemistry 247, 805-814.

Hardy SJS (1975) The stoichiometry of the ribosomal proteins of Escherichia coli. Molecular and General Genetics 140, 253-274.

Harms JM, Wilson DN, Schluenzen F, Connel SL, Stachelhaus T, Zaborowska Z, Spahn CMT and Fucini P (2008) Translation regulation via L11: molecular switches on the ribosome turned on and off by thiostrepton and micrococcin. Cell 30, 26-38.

Heimark RL, Hershey JW and Traut RR (1976) Crosslinking of initiation factor IF2 to proteins L7/L12 in 70S ribosomes of Escherichia coli. Journal of Biological Chemistry 251, 7779-7784.

Helgstrand M, Mandava CS, Mulder FA, Liljas A, Sanyal S and Akke M (2007) The ribosomal stalk binds to translation factors IF2, EF-Tu, EF-G and RF3 via a conserved region of the L12 C-terminal domain. Journal of Molecular Biology 365, 468-479.

Heller JLE, Kamalampeta R and Wieden H-J (2017) Taking a step back from back-translocation: an integrative view of LepA/EF4's cellular function. Molecular and Cellular Biology 37, e00653-16.
Huang C, Mandava CS and Sanyal S (2010) The ribosomal stalk plays a key role in IF2-mediated association of the ribosomal subunits. Journal of Molecular Biology 399, 145-153.

Ilag LL, Videler H, McKay AR, Sobott F, Fucini P, Nierhaus KH and Robinson CV (2005) Heptameric (L12) 6 /L10 rather than canonical pentameric complexes are found by tandem MS of intact ribosomes from thermophilic bacteria. Proceedings of the National Academy of Sciences USA 102, 8192-8197.

Kaltschmidt E and Wittmann HG (1969) Ribosomal proteins: VI. Preparative polyacrylamide gel electrophoresis as applied to the isolation of ribosomal proteins. Analytical Biochemistry 36, 401-412.

Kaltschmidt E and Wittmann HG (1970) Ribosomal proteins. XII. Number of proteins in small and large ribosomal subunits of Escherichia coli as determined by two-dimensional gel electrophoresis. Proceedings of the National Academy of Sciences USA 67, 1276-1281.

Kischa K, Möller W and Stöffler G (1971) Reconstitution of a GTPase activity by a 50 S ribosomal protein from E. coli. Nature New Biology 233, 62-63.

Koteliansky VE, Domogatsky SP and Gudkov AT (1978) Dimer state of protein L7/L12 and EF-G dependent reactions on ribosomes. European Journal of Biochemistry 90, 319-323.

Kravchenko O, Mitroshin I, Nikonov S, Piendl W and Garber M (2010) Structure of a two-domain $\mathrm{N}$-terminal fragment of ribosomal protein L10 from Methanococcus jannaschii reveals a specific piece of the archaeal ribosomal stalk. Journal of Molecular Biology 399, 214-220.

Kumar V, Chen Y, Ero R, Ahmed T, Tan J, Li Z, Wong AS, Bhushan S and Gao YG (2015) Structure of BipA in GTP form bound to the ratcheted ribosome. Proceedings of the National Academy of Sciences USA 112, 10944-10949.

Lake JA (1976) Ribosome structure determined by electron microscopy of Escherichia coli small subunits, large subunits and monomeric ribosomes. Journal of Molecular Biology 105, 131-139.

Langen R, Schweins T and Warshel A (1992) On the mechanism of guanine triphosphate hydrolysis in ras p21 proteins. Biochemistry 31, 8691-8696.

Leijonmarck M and Liljas A (1987) Structure of the C-terminal domain of the ribosomal protein L7/L12 from Escherichia coli at $1.7 \AA$. Journal of Molecular Biology 195, 555-579.

Leijonmarck M, Eriksson S and Liljas A (1980) Crystal structure of a ribosomal component at 2.6 ̊̊ resolution. Nature 286, 824-826.

Liljas A (1982) Structural studies of ribosomes. Progress in Biophysics and Molecular Biology 40, 161-228.

Liljas A (1991) Comparative biochemistry and biophysics of ribosomal proteins. International Review of Cytology 124, 103-136.

Liljas A and Ehrenberg M (2013) Structural Aspects of Protein Synthesis. 2nd Edn. Singapore: World Scientific, ISBN 978-9814313209.

Liljas A, Kirsebom LA and Leijonmarck M (1986) Structural studies of the factor binding domain. In Structure, Function and Genetics of Ribosomes. B Hardesty and G Kramer (eds), New York: Springer-Verlag, pp. $379-390$.

Liljas A, Ehrenberg M and Åqvist J (2011) Comment on "The mechanism for activation of GTP hydrolysis on the ribosome. Science 333, 37.

Lin J, Gagnon MG, Bukley D and Steitz TA (2015) Conformational changes of elongation factor $\mathrm{G}$ on the ribosome during tRNA translocation. Cell 160, 219-227.

Maki Y, Hashimoto T, Zhou M, Naganuma T, Ohta J, Nomura T, Robinson CV and Uchiumi T (2007) Three binding sites for stalk protein dimers are generally present in ribosomes from archaeal organism. Journal of Biological Chemistry 282, 32827-32833.

Mandava CS, Peisker K, Ederth J, Kumar R, Ge X, Szaflarski W and Sanyal S (2012) Bacterial ribosome requires multiple L12 dimers for efficient initiation and elongation of protein synthesis involving IF2 and EF-G. Nucleic Acids Research 40, 2054-2064.

Moazed D, Robertson JM and Noller HF (1988) Interaction of elongation factors EF-G and EF-Tu with a conserved loop in 23S RNA. Nature 334, 362-364.

Mohr D, Wintermeyer W and Rodnina MV (2002) GTPase activation of elongation factors $\mathrm{Tu}$ and $\mathrm{G}$ on the ribosome. Biochemistry 41, 1252012528.

Mondal D and Warshel A (2018) EF-Tu and EF-G are activated by allosteric effects. Proceedings of the National Academy of Sciences USA 115, 3386-3391. 
Mulder FAA, Bauakaz L, Lundell A, Liljas A, Akke M and Sanyal SC (2004) Conformation and dynamics of protein L12 in solution and on the ribosome. Biochemistry 43, 5930-5936.

Mustafi M and Weisshaar JC (2018) Simultaneous binding of multiple EF-Tu copies to translating ribosomes in live Escherichia coli. mBio 16, e02143-17.

Naganuma T, Nomura N, Yao M, Mochizuki M, Uchiumi T and Tanaka I (2010) Structural basis for translation factor recruitment to the eukaryotic/ archaeal ribosomes. Journal of Biological Chemistry 285, 4747-4756.

Nechifor R and Wilson KS (2007) Crosslinking of translation factor EF-G to proteins of the bacterial ribosome before and after translocation. Journal of Molecular Biology 368, 1412-1425.

Oleinikov AV, Jokhadze GG and Traut RR (1998) A single-headed dimer of Escherichia coli ribosomal protein L7/L12 supports protein synthesis. Proceedings of the National Academy of Sciences USA 95, 4215-4218.

Österberg R, Sjöberg B, Liljas A and Pettersson I (1976) Small angle X-ray scattering and cross-linking study of the proteins from Escherichia coli ribosomes. FEBS letters 66, 48-51.

Österberg R, Sjöberg B, Pettersson I, Liljas A and Kurland CG (1977) Small-angle scattering study of the protein complex of L7/L12 and L10 from Escherichia coli ribosomes. FEBS Letters 73, 22-24.

Pallesen J, Hashem Y, Korkmaz G, Koripella RK, Huang C, Ehrenberg M, Sanyal S and Frank J (2013) Cryo-EM visualization of the ribosome in termination complex with apo-RF3 and RF1. Elife 2, e00411.

Pettersson I and Kurland CG (1980) Ribosomal protein L7/L12 is required for optimal translation. Proceedings of the National Academy of Sciences USA 77, 4007-4010.

Pettersson I, Hardy SJS and Liljas A (1976) The ribosomal protein L8 is a complex of L7/L12 and L10. FEBS Letters 64, 135-138.

Remacha M, Jimenez-Diaz A, Bermejo B, Rodriguez-Gabriel MA, Guarinos E and Ballesta JP (1995) Ribosomal acidic phosphoproteins P1 and $\mathrm{P} 2$ are not required for cell viability but regulate the pattern of protein expression in Saccharomyces cerevisiae. Molecular Cellular Biology 15, $4754-4762$.

Santos C and Ballesta JPG (1994) Ribosomal protein P0, contrary to phosphoproteins $\mathrm{P} 1$ and $\mathrm{P} 2$, is required for ribosome activity and Saccharomyces cerevisiae viability. Journal of Biological Chemistry 269, 15689-15696.

Santos C and Ballesta JP (1995) The highly conserved protein P0 carboxyl end is essential for ribosome activity only in the absence of proteins P1 and P2. Journal of Biological Chemistry 270, 20608-20614.

Sanyal CS and Liljas A (2000) The end of the beginning: structural studies of ribosomal proteins. Current Opinion in Structural Biology 10, 633-636.

Savelsbergh A, Mohr D, Wilden B, Wintermeyer W and Rodnina MV (2000) Stimulation of the GTPase activity state of translation elongation factor G by protein L7/L12. Journal of Biological Chemistry 275, 890-894.

Savelsbergh A, Mohr D, Kothe U, Wintermeyer W and Rodnina MV (2005) Control of phosphate release from elongation factor $\mathrm{G}$ by ribosomal protein L7/12. EMBO Journal 24, 4316-4323.

Schmeing TM, Voorhees RM, Kelley AC, Gao YG, Murphy FV, Weir JR and Ramakrishnan V (2009) The crystal structure of the ribosome bound to EF-Tu and aminoacyl-tRNA. Science 326, 688-694.

Shi WW, Mak ANS, Wong KB and Shaw PC (2016) Structures and ribosomal interactions of ribosome-inactivating proteins. Molecules 21, 1-13.

Shimmin LC, Ramirez C, Matheson AT and Dennis PP (1989) Sequence alignment and evolutionary comparison of the L10 equivalent and L12 equivalent ribosomal proteins from archaebacteria, eubacteria, and eukaryotes. Journal of Molecular Evolution 29, 448-462.

Simonetti A, Marzi S, Billas IM, Tsai A, Fabbretti A, Myasnikov AG, Roblin P, Vaiana AC, Hazemann I, Eiler D, Steitz TA, Puglisi JD, Gualerzi CO and Klaholz BP (2013) Involvement of protein IF2 N domain in ribosomal subunit joining revealed from architecture and function of the full-length initiation factor. Proceedings of the National Academy of Sciences USA 110, 15656-15661.

Stark H, Rodnina MV, Wieden HJ, van Heel M and Wintermeyer W (2000) Large-scale movement of elongation factor $\mathrm{G}$ and extensive conformational change of the ribosome during translocation. Cell 100, 301-309.
Stöffler G, Hasenbank R, Bodley JW and Highland JH (1974) Inhibition of protein L7/L12 binding to $50 \mathrm{~S}$ ribosomal cores by antibodies specific for proteins L6, L10 and L18. Journal of Molecular Biology 86, 171-174.

Strycharz WA, Nomura M and Lake JA (1978) Ribosomal protein L7/L12 localized at a single region of the large subunit by immune microscopy. Journal of Molecular Biology 126, 123-140.

Subramanian AR (1975) Copies of proteins L7 and L12 and heterogeneity of the large subunit of Escherichia coli ribosomes. Journal of Molecular Biology 95, 1-8.

Tanzawa T, Kato K, Girodat D, Ose T, Kumakura Y, Wieden HJ, Uchiumi T, Tanaka I and Yao M (2018) The C-terminal helix of ribosomal $\mathrm{P}$ stalk recognizes a hydrophobic groove of elongation factor 2 in a novel fashion. Nucleic Acids Research 46, 3232-3244.

Tchorzewski M (2002) The acidic ribosomal P proteins. International Journal of Biochemistry and Cellular Biology 34, 911-915.

Tchorzewski M, Boguszewska A, Dukowski P and Grankowski N (2000a) Oligomerization properties of the acidic ribosomal P-proteins from Saccharomyces cerevisiae: effect of P1A protein phosphorylation on the formation of the P1A-P2B heterocomplex. Biochimica et Biophysica Acta 1499, 63-73.

Tchorzewski M, Boldyreff B, Issinger O and Grankowski N (2000b) Analysis of the protein-protein interactions between the human acidic ribosomal P-proteins: evaluation by the two hybrid system. International Journal of Biochemistry and Cellular Biology 32, 737-746.

Terhorst C, Wittmann-Liebold B and Möller W (1972) 50-S ribosomal proteins. Peptide studies on two acidic proteins, A 1 and A 2, isolated from 50-S ribosomes of Escherichia coli. European Journal of Biochemistry 25, 13-19.

Tourigny DS, Fernández IS, Kelley AC and Ramakrishnan V (2013) Elongation factor $\mathrm{G}$ bound to the ribosome in an intermediate of translocation. Science 340, 1235490.

Traut RR, Dey D, Bochkariov D, Oleinikov AV, Jokhadse GG, Hamman BD and Jameson DM (1995) Location and domain structure of Escherichia coli ribosomal protein L7/L12: site specific cysteine crosslinking and attachment of fluorescent probes. Biochemistry and Cell Biology 73, 949-958.

Tritton TR (1980) Proton NMR observation of the Escherichia coli ribosome. FEBS Letters 120, 141-144.

Uchiumi T, Wabha AJ and Traut RR (1987) Topography and stoichiometry of acidic proteins in large ribosomal subunits from Artemia salina as determined by crosslinking. Proceedings of the National Academy of Sciences USA 84, 5580-5584.

Valle M, Zavialov A, Li W, Stagg SM, Sengupta J, Nielsen RC, Nissen P, Harvey SC, Ehrenberg M and Frank J (2003) Incorporation of aminoacyl-tRNA into the ribosome as seen by cryo-electron microscopy. Nature Structural Biology 10, 899-906.

van Agthoven A, Maassen JA, Schrier PI and Möller W (1975) Inhibition of EF-G dependent GTPase by an aminoterminal fragment of L7/L12. Biochemical and Biophysical Research Communication 64, 1184-1191.

Voorhees RM, Schmeing TM, Kelley AC and Ramakrishnan V (2010) The mechanism for activation of GTP hydrolysis on the ribosome. Science 330, 835-838.

Wahl MC and Möller W (2002) Structure and function of the acid ribosomal stalk proteins. Current Protein \& Peptide Science 3, 93-106.

Wahl MC, Bourenkov GP, Bartunik HD and Huber R (2000) Flexibility, conformational diversity and two dimerization modes in complexes of ribosomal protein L12. EMBO Journal 19, 174-186.

Wawiórka L, Molestak E, Szahwai M, Michalec-Wawiórka B, Molon M, Borkiewicz L, Grela P, Boguszewska A and Tchórzewski M (2017) Multiplication of P-stalk proteins contributes to the fidelity of translation. Molecular Cell Biology 37, e00060-17.

Wool IG, Chan YL, Gluck A and Suzuki K (1991) The primary structure of rat ribosomal proteins $\mathrm{P} 0, \mathrm{P} 1$, and $\mathrm{P} 2$ and a proposal for a uniform nomenclature for mammalian and yeast ribosomal proteins. Biochimie 73, 861-870.

Yusupov MM, Yusupova GZ, Baucom A, Lieberman K, Earnest TN, Cate JH and Noller HF (2001) Crystal structure of the ribosome at $5.5 \AA$ resolution. Science 292, 883-896.

Zinker S and Warner JR (1976) The ribosomal proteins of Saccharomyces cerevisiae. Phosphorylated and exchangeable proteins. Journal of Biological Chemistry 251, 1799-1807. 